\title{
Method for Performance Analysis of Production Lines Based on Digital Models Powered by Real-Time Data
}

\author{
Suewellyn KRÜGER ${ }^{1}$, Samuel Henrique WERLICH and Milton BORSATO \\ Federal University of Technology - Paraná (UTFPR), Curitiba-PR, Brazil
}

\begin{abstract}
The intention to improve the performance of the processes involved is considered one of the main guidelines for companies' competitive advantage. Technological advances help to improve yields, operations, decision making and cost reduction when adopted in their daily processes, and assembly lines are constantly monitored, but the detection of adversities is still considered complex. Because of this difficulty, the proposal for a virtual model with greater fidelity to reality, such as the digital twin model, is promising. A virtual model of the shop floor system composed of physical characteristics present in the industrial environment, being fed with data that occurs in real-time, offers the simulation possible predictions of setbacks with greater fidelity. This monitoring provides the user with an improvement in the process control, assisting in the planning of operations, enabling changes in the virtual model of compliance verification, improving performance and productivity, reducing related times and costs for later application in the physical model. In this way, the objective of this work proposes the elaboration of an application method where it is possible to analyze the performance of productive lines through digital models integrated with the data feed system in real-time.
\end{abstract}

Keywords. Digital Twin, Industry 4.0, Simulation, Real-time data, Manufacturing System, Transdisciplinary.

\section{Introduction}

The highlight of many companies in relation to their competitors is linked to the use of competitive intelligence, helping in the process of innovating manufactured products as well as improving quality and reducing the costs involved in the procedures performed. Among the various technological advances present in the current market, the involvement of information, equipment and the communication of the entire system in real-time provide an agile and independent data analysis of both the process and the product, advising decision-making in the stages of product development.

In a transdisciplinary way, Industry 4.0 is related by its involvement in several areas such as information technologies, data exchange, the use of integrated systems, the use of the internet of things, cloud computing, modeling and information security. A smart factory based on Industry 4.0 involves a connection between mobility, structures, logistics and networks. Getting decision-making efficiently and in real-time through the

\footnotetext{
${ }^{1}$ Corresponding Author, Mail: suewellynkruger@alunos.utfpr.edu.br.
} 
joining of various technologies with the latest innovations serves as an incentive to practice Industry 4.0 [1].

The automotive industries use the concepts of Industry 4.0 because they consider productivity as central feasibility for the organization, featuring lean manufacturing characteristics, emphasizing the identification and elimination of waste constantly, aiming to reduce delivery times in the processes, improving the management of stocks through faster responses to market changes [2].

In line with Industry 4.0, the use of tools and techniques for monitoring and supervising industrial performance is associated with a competitive advantage. The use of predictive production techniques helps in the search for control of productivity loss points, enabling the anticipation of possible failures and even malfunctions of machines, which can be applied in several areas such as maintenance, planning, programming and even production [3 ].

A technology that can be considered when talking about monitoring is the digital twin, as it is a virtual representation of all the resources in the system. Ranging from the machine and shop floor layout, to the products, processes and tasks performed, entering the real times of the system, thus obtaining a continuous view of the functioning of the entire process.

The use of technologies based on simulations draws even more attention from companies as they allow the demonstration of possible solutions in the virtual environment before applying them to the physical itself. The great related value is in the fact that the user can experiment and evaluate in the virtual certain changes and combinations of factors present in the physical environment system [4].

Always present wishes in the automotive industry involve intelligent manufacturing and a way to assist this is through the virtual modeling of the system. Thus it provides companies with certain preventions, improvement and control of the processes carried out and the simultaneous monitoring of the activities carried out, assisting in the decision making of problems that may occur related to productivity [5].

Through a bibliographic survey, some points were related to support this research. When the search for the term system monitoring in real-time was carried out, little research was found involving the application of digital twin with industrial environments. If the search directs to the association between devices, with sensors and actuators, with the monitoring of the states of the connected devices through the Internet of Things (IoT) in a short period, what is found are only simulations, desiring its application in a real environment for a better evaluation of the method as well as the creation of a human-machine interface for its proper follow-up and monitoring [6].

Having some aspects observed in this bibliographic survey, this research is directed to the difficulties that many companies face in managing to improve performance issues of their systems and to adapt quickly to the production of a new item. The development of a method presented in this article will help to improve the company's flexibility and the difficulty in adapting to the exceptional.

Through the bibliographic survey carried out, it was observed the lack of applications in the practice of a digital model with the feeding of real data in real-time and with the monitoring in real-time, a gap that will be directed to this research. The rest of this work will be structured as follows: section 1 presents the main concepts on the relevant topics involved such as manufacturing systems, digital twin and assembly line. Section 2 describes the entire methodology for proposing the application of the digital model and finally, in section 3, the next steps for the physical application of the model 
and some preliminary results already obtained in the initial stages of the research and information for its continuity.

\section{Background}

In view of the wide possibility of using technologies to virtualize the resources of the processes present in manufacturing, a bibliometric study was carried out and the most relevant scientific productions were analyzed, thus obtaining the edge of knowledge for the themes involved with this research.

A pointed trend [1] is the implementation of digital twin through a structural system with all the elements connected. Making its modularity, autonomy and connectivity possible through installations and the use of sensors and actuators distributed by the system.

The need to use current software is presented [7] as a challenge for the rapid updating of data, such as the times of individual cycles and the degrees of use of workstations and robots. In addition, [8] the application of a digital twin model for production control in a continuous manner and updated in real-time must reflect exactly what is happening in the physical environment, allowing for optimizations, quick and concise decision making, intervening in a predictive manner.

A relevant point pointed out in another research [6] indicates that it is essential to detail the modeling concepts for more complex environments when analyzing the Internet of Things (IoT) devices, such as the industrial factory floor. Modeling tools often do not support simulation and analysis due to the high number of representations required in the virtual model of equipment such as machines, devices, sensors and actuators.

When the digital twin model is directed to the shop floor, it considers its own research [9] as an initial stage and points to future work the interconnection and intelligent interaction of the shop floor, a bidirectional connection between the physical and virtual environments, perfecting the virtual model with the increase of fidelity are fundamental for future applications of the digital twin shop-floor in intelligent fabrications.

The involvement of Industry 4.0 in a transdisciplinary way adds value to the client by presenting the real conditions of the process, with a high number of information implemented in the simulation, bringing the virtual representation closer to reality, obtaining more efficiently the indication of possible problems in the project and directing appropriate improvements in advance.

To demonstrate the main issues related to this research, the following will present relevant concepts and approaches found in reputable scientific publications. Firstly, it will be discussed on manufacturing systems, followed by an approach regarding digital twin and finally on production lines with a focus on performance analysis, flexibility and adaptability.

\subsection{Manufacturing System}

The manufacturing systems are constantly improving so that they can respond to the involvement of new technologies, quickly and efficiently directing their final objective, providing opportunities for the activities involved and improving the final quality.

In general, manufacturing systems can be seen as a set of complex activities because they involve people, a high diversity of machines and tools, as well as other equipment 
in its various levels of automation, such as robots and computers. A manufacturing system starts with the development of a product, proceeding to the manufacturing engineering, composing the development of the production processes, as well as the planning of the necessary resources for the effective production of the product and its delivery to the customer [10].

This whole set of activities and operations is associated with the necessary inputs, the transformation processes and the manufacturing life cycle. Depending on the degree of complexity of these variables, robust monitoring has great value, allowing the whole process to occur in the best possible way [11].

More and more [12] companies are inserting digital and intelligent manufacturing in their procedures, improving the modeling of design data, manufacturing, management and maintenance of the operations involved.

When all this transdisciplinary involvement is analyzed, there is a need for research regarding the involvement in data capture in an automated way and the generation of a database in real-time. Connecting the physical and real systems allowing access, exchanges and data processing, collaborating in the manufacturing systems and boosting Industry 4.0, we can mention the cyber-physical systems (CPS) [13].

Following the architecture of CPS with Industry 4.0 [14], the proposal of the five levels in the systems provides an intelligent analysis for integrity, allowing the modeling of the digital twin and assists in decision making. Working its connectivity in an advanced way in the collection of data in real-time of the physical environment with the management, analysis and computation of the data in a cybernetic environment.

When PHCs are used [13], aspects such as self-organization, security, remote diagnosis, real-time control, transparency, predictability, efficiency and possible corrections in the virtual model are expected to be improved. All these aspects add value to an organization based on Industry 4.0 by presenting autonomous optimization, promoting the digitization of the entire production, in addition to the involvement of workers, products and resources with intelligent instances.

Researches consider CPS as promising for its involvement with IoT, through the transdisciplinary distribution between the resources of computing, communication and control of physical systems, not to mention its use as a data provider for digital models. These models represent resources of the process assisting in decision making and production planning, because through their analysis actions are possible in the physical environment, such as exchanging information providing an improvement in the quality of services and suggesting in the proposal in the use of digital twin in a manufacturing process.

\subsection{Digital Twin}

A digital twin model can be seen as a set of virtual information to portray a product, a system or a physical process. From the minimum level of the machines, through the factory level as the assembly line, to the maximum possible as the organizational level for example [15]. Having been seen as a new technology inserted in the industrial environment, the analysis of the effects caused in the most complex scenarios present and its monitoring in decisions over time is essential.

When related to manufacturing, the digital twin can be defined as a virtual representation of the production systems to synchronize the virtual and real systems. This synchronization can be carried out [8] using connected smart devices allowing the 
communication of data in real-time, the basis of Industry 4.0 with its involvement of technological resources for the prevention and optimization of recurrent behaviors in the system.

Its use in manufacturing also makes it possible to simulate and optimize production systems as it encompasses several aspects, providing a visualization of the entire process, whether at the level of just one component or even the entire assembly line [16]. Emphasizing the areas of control, production planning, maintenance and also layout planning, aiming at increasing competitiveness. The digital twin is seen with great potential in planning and optimization simulations [9], in increasing efficiency and precision, obtaining considerable financial gains for production [8], and also because it is not just a large collection of digital artifacts, but a structured model with all the elements connected [1].

The digital twin model emphasizes the control and decision-making of processes due to the identification of deviations from the current operation of the equipment and its causes, helping to identify possible emergencies and generating alerts for the monitoring team about abnormal events and situations [17]. Among the advantages of its implementation, we can mention the use of production equipment with mechanisms and operations monitored by devices. The collection of the generated data takes place through sensors, actuators and optimized software.

Different levels of integration between the physical and virtual models are defined according to the modeling. A level can be a digital model when there is no automated exchange of data between the models, always taking place manually. It can also be a digital shadow when the automated data flow is unilateral, and any change in the physical environment has repercussions in the virtual, but the opposite path does not occur. And finally, the digital twin, in which the data exchange flow occurs in both directions, providing control on both sides, allowing interventions in the plant, physical environment, through the virtual environment, its executions and the responses of the physical environment being obtained in the virtual [16].

Therefore, the application of the digital twin in an industrial environment includes the improvement of situational knowledge of the entire process with the insertion of the real data of the system, enabling the improvement in the responses of the conditions of possible changes and optimization of future conditions with the permission of analysis of performing predictive maintenance in the process of production lines, such as automotive assembly lines.

\subsection{Assembly line}

There are several cases of application of digital twin in manufacturing processes, so there is an opportunity to apply it on an assembly line. The assembly lines can also be called progressive assembly precisely because there are associations and joints of parts as they travel through the workstations distributed on the factory floor, being sequentially incorporated one by one until the final product.

The execution of similar methods in the production of items such as automobiles, the transportation of equipment and the manufacture of household and electronic devices are considered important characteristics for an assembly line. As well as the sequential disposition of those involved in it, as operators and tools, as well as the machines and parts, used to produce the product.

The layout of an assembly line can be considered a direct influence on productivity, allowing for several dimensioning configurations for the line [18]. Another important 
feature is the access to the parts that will be used coming to the work stations, their displacement occurs by transporters or motor vehicles.

The complexity of the assembly lines is due to the high number of interconnected variables for their operation. These variables are related to technologies, environmental factors, ergonomic issues and the methods and times for the production of parts [19].

As soon as an assembly line is drawn up, the first characteristic that must be analyzed is its balance. For the construction of this line, some points must be considered, such as the verification of all the essential steps to manufacture each item of the product, the balance of the procedures along the line must be measured, the number of machines and their production rates is also important.

In an automotive assembly line, all components must be integrated sequentially as the product will be formed and transported by a transporter. As the product passes through the line, each operator performs a specific task for their workstation by placing supplies such as parts and tools at their point sequentially. The company's flexibility in adapting and incorporating new activities in the manufacturing process and in adapting to changes in actions and in the production flow quickly and effectively are important factors for a good manufacturing process.

\section{Methodology}

There are promising conditions for companies that are involved with the use of competitive intelligence. The use of knowledge involving intelligent manufacturing with the technologies of industry 4.0, as a way of assisting decision-making in production processes, whether new or existing, promotes the improvement of solutions for applications in production lines with higher quality.

The direction of this research relies on selected literature for a diagnosis of the problem in question and through observations on the procedures performed in the company of the application case. This investigation took place through interviews with the parties involved as process and project engineers together with the analysis of the documents and times for the procedures to be carried out.

The observation of the procedures and tasks carried out in the company were analyzed and resulted in the proposal of an analysis of the performance of the production line aiming at the reduction of costs involved with the detected problems. These problems may be related to the lack of a virtual model to enable checks and compliance with changes in the physical environment of the production line.

Changes to an existing project or the insertion of a new product on an assembly line, cause the responsible team to spend a lot of time between trips, attempts, errors and successes so that the job can be left with optimum performance. Because of this issue, a digital model was chosen. Therefore, this research aims to develop an application method to analyze the performance of the production lines, through a digital model fed with real data and in real-time providing simulation of ideas in a production environment.

The simulation provides an advantage for its virtual implementation. The team can have a vision of the assembly line and create scenarios in advance, because until then this task would only be possible after the complete definition of the project, resulting in numerous subsequent changes generating a high rate of rework. The simulation assists in boosting innovation so that it is possible to virtually carry out definitions, plans, creations, monitoring and control of all production processes. 
The application of digital twin in the simulation of processes provides the company with greater security in decision making by presenting efficient monitoring and aptitude to the actions performed physically, increasing mobility, enabling the user to make changes and monitor remotely, without interfering and/or cause losses in the results obtained by the production. The balancing of the company's assembly line can be used to promote an increase in income, a reduction in the costs involved, thus increasing the profits generated. In addition, activities are sequentially distributed among workplaces according to their operating times, improving the use of operations as well as the equipment present.

When implemented in digital manufacturing, the use of digital twin virtually integrates design information and manufacturing processes. Aiming at reducing the costs involved in the development, anticipating and non-conformities of the system, providing innovation in the manufacturing area with higher quality products, allowing the creation and application of innovative models with their due representations and also with the simulation of possible scenarios in the search for optimization.

Another reason for the use of the digital twin model in this research is because it does not deal only with the design and optimization of a production system, but involves involving real data from the production environment, continuously offering a simulation of the system and contributing quickly in real-time feedback throughout the manufacturing process [20]. Some steps must be taken so that the proposed method can reach its final objective, as shown in Figure 1.

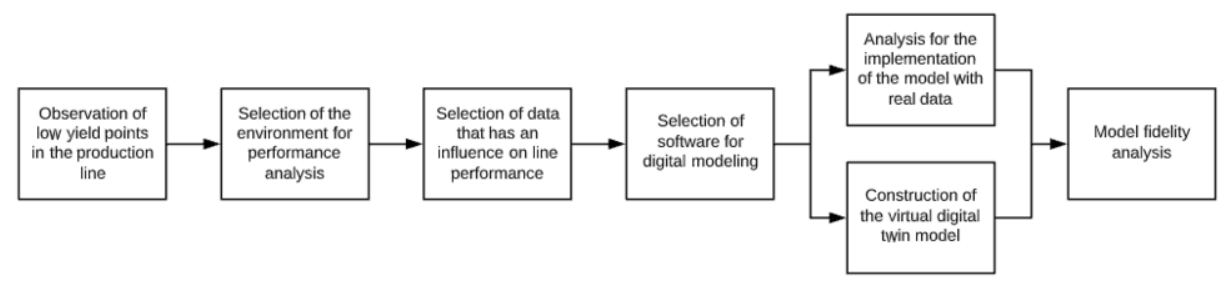

Figure 1. Performance analysis method.

Using the method developed for the performance analysis, observations were first made of the points that face problems in the assembly line. These points result in low yield, high index with failures in quality, low in capacity and gains in production. Through the analysis of a monitoring system present in the company of the application case, historical data was extracted from points where there were large numbers of line stops and directed to points with low performance.

In the next stage, an environment was selected to perform the performance analysis. A fraction of the assembly line that will be virtualized was selected since it does not have the full operation of the equipment involved in the procedures, it has considerable levels of non-quality of the final products and a large volume of rework.

Another fundamental point for choosing this cut was that this line has more automated factors and characteristics than the others, thus presenting greater conditions for the implementation of the digital twin model. This fraction of the assembly line can be considered complex due to the number of functions and actions performed, in addition to all the sequencing of activities performed by machinery present. This cutout corresponds to the part of the line where the wheels are mounted for their subsequent insertion in the vehicle. 
After choosing the point to be virtualized, the selection of data to feed the digital model is essential. Data such as the type of wheels produced, the number of wheels audited, the number of wheels in compliance, as well as in non-compliance are some of the important data extracted from the devices of the line. Other relevant data for the virtual model are the factory and line layout, downtime and setup times, operating times and maintenance schedules. These data directly influence the performance of the line, and other data may also be collected for the construction of the virtual model.

The software to be chosen for the modeling must be available in the company in the case of an application for the implementation of the data in real-time, directly with data from the assembly line. The line data is recorded in a MOP (Marge OPérationnelle), prepared using the Node-Red tool based on the communication flow from the hardware devices to an online environment and then sent via the UDC protocol (User Datagram Protocol) to Spotfire, responsible for carrying out intelligent data analysis. Finally, the work and structured data will be sent to the GCP cloud (Google Cloud Platform).

The storage and processing of the information will be carried out in the GCP resulting in a dashboard with the relevant information for the virtual model. Other important data for the virtual model will be inserted in the simulation through interfaces present in the simulation software.

After having created the virtual model and the relevant data being fed, a fidelity analysis must be carried out to verify compliance. Observations of what happens in the digital model will be made according to what happens in the real environment, resulting in coherent replicas. Thus, this virtual model can be accepted for the proper continuation of the research. When changes in the virtual model are applied to the physical environment, observing its occurrence requires attention by comparing the results obtained through the virtual simulation in accordance with what occurs at the end of physical production.

\section{Results/ Conclusion}

Therefore, through the procedures demonstrated in this research, the method proposed in an organizational environment will seek an estimate of the realization of the processes, aiding in the prevention of possible mistakes and aiding in the company's decisionmaking in the case of application. The provision of a digital manufacturing process environment is conducive to the optimization of production systems and processes.

The digital twin is being carried out in several types of research recently and its targeting for the manufacturing area collaborates with the production planning and control. The use of real data in the virtual model with real-time feed is considered promising for the improvement of its application in production systems.

Another important point for the elaboration of this digital twin model is to make interventions on the assembly line via distance, avoiding that there are comings and goings to the factory floor to observe, analyze and, mainly, cause a line stop to perform some attempted change. These unnecessary displacements result in activities that do not add value to the company and unplanned line stops have an impact on production and quality losses.

Some data from the wheel production machines, which will be inserted in the virtual model, have already been pre-determined because they are considered essential for a simulation, such as the operating times, the line stop times, the average cycle times and the causes of line stop for predictive analysis. Other data will still be evaluated for 
insertion or not in the virtual model, always in the search to increase the degree of fidelity.

The realization of this research has some risks that must be taken into account, which may result in its planning. As your application will be carried out in a company in the automotive industry, you must have full access to information and data related to the production line. In addition to the data, access to the company's system as well as to the available software that is compatible with what is wanted.

Among the positive points, it can be considered that this project will bring gains for the company in the application case, by doing virtual modeling of the production line and mainly simulating with data inserted in real-time, making it possible to boost business competitiveness. With the mirroring of the virtual model, with what is happening in the physical environment, they support production planning and monitoring, reducing production downtime, impacting the costs involved with maintenance in addition to the increase in productivity resulting in profits for the company.

\section{Acknowledgement}

Special thanks to the Fundação Araucária for encouraging research.

\section{References}

[1] R. Rosen, G. Wichert, G. Von and G. Lo, About the importance of autonomy and digital twins for the future of manufacturing; IFAC-PapersOnLine, 2015, Vol 28, pp. 567-572.

[2] H. Faye and P. Falzon, Strategies of performance self-monitoring in automotive production, Applied Ergonomics, 2009, Vol. 40, pp. 915-921.

[3] B. R. Ferrer, U. Muhammad, W. M. Mohammed and J. L. M. Lastra, Implementing and visualizing ISO 22400 key performance indicators for monitoring discrete manufacturing systems, Machines, 2018 , Vol. 6,39 .

[4] D. Mourtiz, M. Doukas and D. Bernidaki, Simulation in manufacturing: review and challenges, Procedia CIRP, 2014, Vol. 25, pp. 213-229.

[5] K. Agyapong-Kodua, R. Darlington and S. Ratchev, Towards the derivation of an integrated design and manufacturing methodology, International Journal of Computer Integrated Manufacturing, 2013, Vol. 26, pp. 527-539.

[6] P. Hirmer, U. Breitenbücher and A. C. F. da Silva, Automating the Provisioning and Configuration of Devices in the Internet of Things, Complex Systems Informatics and Modeling Quarterly, 2016, Vol. 9, pp. 28-43.

[7] F. Biesinger, D. Meike, B. Kras and M. Weyrich, A Case Study for a Digital Twin of Body-in-White Production Systems General Concept for Automated Updating of Planning Projects in the Digital Factory, IEEE International Conference on Emerging Technologies and Factory Automation, 2018, pp.19-26.

[8] E. Negri, L. Fumagalli and M. A. Macchi, Review of the Roles of Digital Twin in CPS-based Production Systems, Procedia Manufacturing, 2017, Vol. 11, pp. 939-948.

[9] F. Tao and M. Zhang, Digital Twin Shop-Floor: A New Shop-Floor Paradigm Towards Smart Manufacturing, IEEE Access, 2017, Vol. 5, pp. 20418-20427.

[10] A. Bargelis, G. Hoehne and A. Cesnulevicius, Intelligent functional model for costs minimization in hybrid manufacturing systems, Informatica, Vol. 15, 2004, pp. 3-22.

[11] H. Elmaraghy, T. Aldeddawy, S. N. Samy and V. Espinoza, A model for assessing the layout structural complexity of manufacturing systems, Journal of Manufacturing Systems, 2014, Vol. 33, pp. 51-64.

[12] B. Yang, L. Qiao, N. Cai, Z. Zhu and M. Wulan, Manufacturing process information modeling using a metamodeling approach, International Journal of Advanced Manufacturing Technology, 2018, Vol. 94, pp. 1579-1596. 
[13] L. Monostori, B. Kádár, T. Bauernhansl, S. Kondoh, S. Kumura, G. Reinhart, O. Sauer, G. Schuh, W. Sihn and K. Ueda, Cyber-physical systems em manufacturing, Manufacturing Technology, 2016, Vol. 65, pp. 621-641.

[14] K. M. Alam and A. El Saddik., C2PS: A Digital Twin Architecture Reference Model for the Cloud-Based Cyber-Physical Systems, IEEE Access, 2017, Vol. 5, pp. 2050-2062.

[15] R. Stark and T. Damerau Digital Twin. In: S. Chatti, L. Laperrière, G. Reinhart, T. Tolio (eds.): CIRP Encyclopedia of Production Engineering. second edition, Springer, Berlin Heidelberg, 2019, DOI: 10.1007/978-3-642-35950-7_16870-1.

[16] W. Kritzinger, M. Karner, G. Traar, J. Henjes, and W. Sihn, Digital Twin in manufacturing: A categorical literature review and classification, IFAC-PapersOnLine, 2018, Vol. 51, No. 11, pp. 1016-1022.

[17] K. Ponomarev, N. Kudryashov, N. Popelnukha, and V. Potekhin, Main principals and issues of digital twin development for complex technological processes, Ann. DAAAM Proc. Int. DAAAM Symp., 2017, pp. 523-528

[18] D. Battini, M. Faccio, A. Persona, and F. Sgarbossa, New methodological framework to improve productivity and ergonomics in assembly system design, Int. J. Ind. Ergon., 2011, Vol. 41, pp. 30-42.

[19] F. Caputo, A. Greco, M. Fera, and R. Macchiaroli, Digital twins to enhance the integration of ergonomics in the workplace design, Int. J. Ind. Ergon., 2019, Vol. 71, pp. 20-31.

[20] M. Peruzzini, F. Grandi, and M. Pellicciari, Exploring the potential of Operator 4.0 interface and monitoring, Comput. Ind. Eng., 2020, Vol. 139, 105600. 